\title{
Towards Developing Reusable NLP Dictionaries
}

\author{
Pim van der Eijk and Laura Bloksma and Mark van der Kraan
}

\author{
Research Institute for Ianguage and Speech \\ Foundation for Ianguage Technology \\ State University of Utreclit \\ The Netherlands \\ vandereijk@let.ruu.nl
}

\begin{abstract}
Development of reusable dictionaries for NIIP applications requires a carefully designed lexicological framework, a lexical acquisition strategy, an integrated development toolbox, and facilities to generate dictionaries for client applications. 'This paper presents results of the LExic project ${ }^{t}$, which was set up to prepare the development of large multilingual lexical resources.
\end{abstract}

Keywords: lexicons, tools, large-scale resources, typed feature structures.

\section{Introduction}

\subsection{CoInmon Linguistic Resources}

A large amount of the investments in the development of any NLP application is spent on the construction of what one might call "large databases of lexical and grammatical resources". These resources could in principle be useful for many applications although they hardly ever are: due to the lack of agreement on the definition of basic notions and of consensus on the analysis of linguistic phenomena they are often linked too closely to specific applications. Moreover, given the generally limited size and duration of NI,P projects both quartity and quality of such project-specific databases are disappointing.

In this paper we will discuss results from the LEXIC project, a feasibility study preparing large-scale develop-

\footnotetext{
${ }^{1}$ The Lexic project was financed and supported by the three project partwers: Philips Research, developing the Rosetta machine trandation system, the Foundation for Language Technology, participating in the Eurotra project, and Van Dale, one of the main dictionary publishers in the Netherlands, as well as by the the European Commission, and the Jutch ministries of Education and Vconomic Affairs. Detaits of the project are discussed in [van der Eijk rt al., 1991).

The allthors want to thank Anne van Bolluis, Joy Iferklotz, Jeroen Fokker and Tïm Dumas for contribution to the activities discusted in this paper.
}

ment of a reusable lexical database, started by a comsortiun of industrial and university partuers. The lexical database is designed to consist of an integrated package of two monolingual dictionaries for Dutch and Spanish and the bilingual dictionaries relating these langunges. 'The consortium comprised a dictionary publisher as well as NI, application developers, giving it the unique opportunity of confronting the large body of experience, infrastructure and existing data of publishers with the requirements of a new class of professional ukers.

Another interesting aspect of the project was that it addressed the whole spectrum of issues in lexical database development, from lexical acquisition to serving heterogeneous client applications. In the current abbence of any standard for the (grammatical) content of the dictionary (e.g. standardized sets of grammatical features) the reusability of a dictionary can only he eval. wated in terms of usability for some target applications.

\subsection{Structure of the paper}

Section 2 discusses the issue of acquisition of lexical data. Section 3 introduces the implementation formalism and tools. The lexicon architecture is discussed in section 4. Conversion of data to client applications of the database is discussed in section 5 .

\section{Acquisition}

\subsection{Strategies}

'There are three potentially useful strategies to develop large lexical resources, which are not in principle mutually exclusive.

MRDa 'The extraction of data from machine-readable dictionaries has received much attention in the past decade. In our view the urefulness of existing material for NLI' application has been somewhat overestimated. Traditional dictionaries are oriented towards a market of human consuners, who consult the dictionary for entirely different reasons than NI.P applications. For instance, most of the information in NLP dictio. naries is concerned with the grammatical description of 
words, which in many dictionaries is only rudimentarily available ${ }^{2}$.

Furthermore, given that humans can use their intelligence and knowledge of the language(s), much information is only present in unformalized definitions and examples. As discussed in e.g. [McNaught, 1988], it is often feasible to extract (relatively) formalized information, but the cost-effectiveness of automatic extraction of information from less formalized data is highly questionable.

From this discussion it follows that MRDs alone cannot be the source for NLP dictionaries. In section 2.2 we will discuss in more detail the evaluation of the potential sources of data for our specific purposes.

Corpora Automatic extraction of lexical features by applying various pattern recognition techniques to large bodies of text has received some attention recently (cf. e.g. [Zernik and Jacobs, 1990]). However, the information needed for our applications cannot be extracted from corpora yet, although important improvements can be expected in the following years.

Lexicography Given the present inadequacy of $M R D_{8}$ and corpus-related tools, manual labour is indispensable for lexicon development. The tools described in section 3 have been developed as a 'workbench' to support these lexicographical activities. We will show that this tool allows for easy integration of information extracted from MRDs with lexicographic editing.

\subsection{Sources}

Evaluation Measure It is difficult to assess the "reusability" of existing data without an evaluation measure, i.e, without knowing for what purpose the data should be usable. This is especially difficult in the case of grammatical features. We developed a lexicon fragment (implemented as TFS type hierarchy, cf. section 3) defining the classification scheme for the monolingual dictionaries. This fragment is inspired by HPSG and GB, and incorporates many of the (innovative) distinctions developed by the client applications EuroTra and RosETTA. It is, however, much more lexicalist than these systems.

Eventually, all lexical entries in the two languages should be described using this scheme, so that they can be readily converted to client applications. The data that can be extracted from a potential source has been interpreted with respect to this classification scheme to assess the amount of information contained in it.

Data Arulysis The machine-readable sources we considered are the existing Van Dale Dutch monolingual and bilingual Dutch-Spanish machine-readable dictionary and the Celex lexical datubase. From our evaluation it followed that existing MRDs for Dutch (as for almost all other languages) contain only a small part of the information needed by NLP applications.

\footnotetext{
${ }^{2}$ Well-structured dictionaries like [Longman, 1987] are ant important exception to this, cf. [Boguraev and Briscoe, 1989].
}

Fortunately, the CELEX lexical database has enriched a selection of 30000 entries of the "Van Dale Dictionary of Contemporary Dutch" with grammatical information, taking into account the requirements of a number of (prototype) NLP applications under development in the Netherlands. A large amount of information needed for our target applications can be converted automatically from this database. The entries, stored in a relational database, can be imported into the Dutch lexicon using the TFS constraint solver similarly to the conversion to client applications (see section 5). The Cessx dictionary has historic links to the Van Dale dictionaries (especially with respect to reading distinction), which greatly simplifies integration of these sources.

With respect to translation information we found that the "raw" translational data could be extracted easily from the Van Dale bilingual dictionaries. The original Van Dale concept is especially interesting for multilingual applications, as the Dutch part is the same (at least in principle) in all bilingual dictionaries with Dutch as source language (cf. [van Sterkenburg et al., 1982]).

Extraction of information about phrasal translation, such as the choice of the support verb of a noun in the target language, is unfortunately hidden in unrestricted text (example sentences etc.), from which it is difficult to extract. Phrasal information also suffers greatly from incompleteness.

\section{The TFS Formalism}

Before discussing the proposed lexicon architecture we will introduce the computational framework in which it has been formalized and implemented, the formalism of typed feature structures.

Currently the family of unification-based formalisms is an emerging standard as the implementation formalism of natural language processing systems. A variant called typed feature structures, discussed a.o. in [Carpenter, 1990], [Emele and Zajac, 1990] and [Zajac, 1990], has been adopted in a number of European lexicon projects, including ACQULLEX, EURo'TRA 7 and MULTilex. In the course of our project, a TFS database, user interface and a constraint solver have been implemented.

TFS is an excellent formalism for computational lexicons, as it enables a definition of types, or classes, of linguistic objects, arranged in a multiple inheritance hicrarchy, where types are associated with an appropriateness specification defining their features and the types of those features and with (possibly disjunctive and complex) constraints. The object-oriented character of the systern allows for minimization of redundancy, whereas the type system maximizes integrity of data.

Three TFS-based tools have been developed:

- a tool for interactive definition ${ }^{3}$, entry and modification of data (cf. section 3.1).

- a TFS database which can be accessed from the user interface and the constraint solver.

\footnotetext{
${ }^{3}$ The TFS-editor can be used to interactively define a type hierarchy, as such a hierarchy can be viewed itself as a typed feature structure, cf. [Fokker, 1992].
} 
- a TFS-compiler for data manipulation, e.g. selections and conversion.

The TFS-compiler is similar to the systems described by [Carpenter, 1990], [Emele and Zajac, 1990], and [Franz, 1990], and like these it constitutes a generalpurpose constraint-based formalism which can be used for a wide variety of tasks, including parsing, translation and generation. Our prototype is implemented on top of Sicstus Prolog, and is used primarily for selection and conversion of data. It offers a number of tracing and debugging facilities to assist in the design of typehierarchies and during query-evaluation.

These three tools can import and export data in a special-purpose text format, which is useful for interchange and further processing. The acquisition tools for the Van Dale dictionaries and Celex ctm also generate their output in this format.

\subsection{User Interface}

The hierarchical definition of the grammatical types in TFS corresponds closely to a "decision tree" which the lexicographer traverses while editing a lemmi. A graph. ical user interface has been developed by the computer science department of the State University of Utrecht ([Fokker, 1992]) which allows the user to narrow down the main type of the lemma (s)he is editing to a specific subtype and to subsequently edit the associated feature structure. For example, a lemmi is refined from ENTRY to VERB to DATIVE.VERB, then constraints for this type are retrieved and the features and their substructures can be edited recursively.

Of course, only appropriate features are presented and can be edited, e.g. it is impossible to edit a feature a $\mathrm{rg} 9$ of an intransitive verb. While editing the value of a fea ture the editor creates a subwindow alroarly positioned at the minimal type of this feature. E.g. while editing a verb, the feature semantics will already be positioned at the type EVENT, as this is the minimal type of this feature for verbs.

'The editor includes a useful help facility which can be viewed as an on-line instruction manual: a help function exists for each choice point which describes a number of criteria and examples to help making the decision.

It will now be clear how lexicographic work using the decision tree model relates to importation of lexical data from existing sources, such as MRDs. These cas be converted to partially edited lexical entries, so that the lcxicographer doesn't have to start at the 'root' level (c.g. the choice point ExTRY in the example), but at an intermediate level (e.g. VERB). liurther choices lead to more refined descriptions of the word. Like all errors, errors it the source dictionary can be corrected by moving back to a higher-level choice point in the hierarchy.

Completed entries, and also arbitrary substructures, can be narned and stored in a database for future use as shared (sub)structures in other entries. Useful applications of this cross-reference mechanism are in morphology and for the implementation of synonymy (sce 4.2). Compounds can be assigned a feature tree with features left_daughter and right_daughter, whose values are pointers in the database to their constituting parts.
The editor has been implemented in $C^{\prime}$ using the Microsoft Windows 3.0 graphical interface. The program is designed to be easily portable, e.g. to $\mathrm{X}$ windows. 'The underlying database can be shared via a LAN. As the other tools, the database allows for import and export of feature structures in the interchange format.

The editor is designed specifically for the TPS formalism. However it can be used for any specific type hierarchy, as the definition of the type hierarchy is simply defined in a separate lext file which is read by the program during start up. Hence, it is potentially interesting for the development of rnany other (NLP) dictionaries.

An interesting elaboration of the editor would be to add extra functionality for the lexicographer hesides editing and viewing feature structures, such as facilities to consult various on line dictionaries or text corpora.

\section{Dictionary organization}

Ilaving introduced the computational framework we will proceed witl the discussion of the organization of the dictionary 4 . 'I'he emphasis has been on two types of nodularity

1. Modularity of dictionaries and thesaurus.

The general approach is to define clearly a number of alstraction levels (cf. section 4.1) in order to achieve easy comectability of the monolingual dictionaries via bilingual dictionaries. By generalizing bilingual translation to bilingual synonymy (or equivalence, cl. section 4.2) we can even separate semantic descriptions ("concepts") from the elements in which they are realized in languages. We will show how sucl conceptual dictionaries can be gerterated from bilingual dictionaries (4.3).

2. Modularity of grammatical desciption (cf. section 5).

With respect to the linguistic content of the mono lingual dictionaries (i.e. Lhe grammatical descrip)tion) we will diseuss the use of typed feature structure constraints expressing relations between granmatical descriptions in various linguistic theories. This allows for a very flexible relation hetween varjous grammatical descriptions.

\subsection{The nomolingual dictionary}

Word forms in a linguage, as found in text corpora, are associated with canonical forms according to lexi. cological conventions. In prarticular contexts they are associated with exactly one of a fixed finite number of designations". In [Kgustin, 1971], two other "compo nents" of ineaning are distinguished besides designation, viz. connolation and range of application. Our (somewhat poor) working definition of synonymy is a relation between readings sharing designation only, both within a language and across languages (where it is traditionally called equivalence).

T'This is a condensed summary of [van der lijjk, 1992a].

${ }^{5}$ Note that we adopt the approach of discrete readings, $\mathrm{cf}$. [ten Hacken, 1990]. 
The relation between word forms and canonical forms is many-to-many: orthographic variants are mapped onto a single canonical form, and a single word form can be related to several lexical entries via inflectional rules 6 . The monolingual dictionary is a set of lexical entries, which are pairings of canonical word forms of a language and their designations, and in addition describe theit grammatical properties.

As a result, a lexical entry should minimally have the two features canonicalform and semantics. The former feature has the simple type STRING, the latter, the description of the designation, has a complex value, possibly including semantic features, but minimally containing an identifying feature ${ }^{7}$, as we want to make sure it will always be possible to interconnect the monolingual dictionaries via bilingual dictionaries. Apart from these two features, there will be other features for the description of the grammatical properties of the word.

The combination of canonicalform and grammatical description should allow for the complete and correct. generation of all word forms and their associated feature structures. As our intended client applications lave front ends for this purpose the database was not designed to be a full form dictionary; this could change, depending on the needs of future client applications.

The set of designations can be viewed as a thesaurus or "knowledge base"; the lexical entries are "pointers" from words into this knowledge base, and can be implemented as such in TFS.

The relation between canonical word forms and designations is also many-to-many, due to synonymy (scveral word forms related to the same designation) and lexical ambiguity (one word form related to several designations). In addition to this there will be alternations in the description because of alternative grammatical patterns. These alternations are implemented as TFS disjunctions.

\subsection{The bilingual dictionary}

Bilingual dictionaries can be viewed as a relation between words in two languages. The levels "word form", "lexical entry" and "reading" correspond to various degrees of granularity in bilingual dictionaries. Ideally, the bilingual dictionary relates lexical items between languages at the level of readings, though in practice most existing dictionaries refer to canonical forms or even to word forms in the target language. Furthermore, the source language side in bilingual dictionaries usually refers to readings different from the monolingually motivated ones, because they are tuned to the target language: two readings are not distinguished if they trallslate to the same word, or an additional reading is created for an additional translation. An exception is the original concept of the bilingual Van Dale dictionaries, where the source language reading structure of the bilingual

\footnotetext{
${ }^{6}$ F.g. the Dutch word form bekend is associated with the adjective bekend (meaning well-known) and (by participle formation) to the verb bekennen (to confess).

The name of atored semantic substructures in the I'TS database serves this purjose.
}

dictionaries is baned directly on the monolingual reading structure (cf. [van Sterkenburg el al., 1982]).

An interesting approach to the bilingual dictionary would be to view it as dexcribing pairings of bilingual synonyms. The advantage of this would be that

1. the dictionary supports preservation of meaning in translation.

2. formal properties of equivalence relations (e.g. transitive closure) cun be exploited to automatically expand the dictionary

3. coding efforts can be reduced: the definition of the designation can be shared between monoliugual and bilingual synonyus.

The main difference between truditional dictionuries and our approach is therefore that the indirect translational description of bilingual synonymy is replaced by a divect relation between lexical entries in the monolingual dietionaries to an independent "knowledge base" of synonym clusters. This appronch is common in e.g. multilingual terminology (ct. [I'icht and Draskau, 1985]), but less common in lexicology.

We will show that the two representations can be translated into each other. Section 4.3 describes how a knowledge base is generated from monolingual and bilingual dictionaries. A bilingual dictionary can be generated automatically from a set of monolingual dictionaries and a knowledge base by enumerating the pairs of lexical entries in two monolingual dictionaries pointing to the same synonym cluster.

\subsection{Generating Synonym Clusters}

Existing machine-readable bilingual dictionaries ${ }^{8}$ can be converted to a representation based on bilingual synonymy, by "extracting" the underlying concepts. The process consists of the following steps:

First, the dictionaries are parsed and transformed to a table symonym of the relation between a reading $R_{1}$ in a language $L_{1}$ and a reading $R_{2}$ in $L_{2}$. Two versions of this program have been developed and tested: one for the Van Dale Dutch-Spanish dictionary and one for bilingual entrics in the EUROTRA transfer rule format. A version for dictionaries in a standard interchange format would be a possible future extension.

Second, reflexive, symmetric, and transitive closure is applied to the symonym $/ 4$ relation ${ }^{9}$. For each reading the generated synonym cluster can be viewed. E.g. according to the Van Dale Dutch-Spanish dictionary, reading 0.1 of Dutch eerbetoon (English (mark of) honour) has one synonymous reading in Dutch and three synonyms in Spanish.

enrbetoon_0.1:

${ }^{6}$ Actually, there is no restriction to a blingual dictionary: several bi- or multilingual dictionaries, and even monolingual dictionarice of synonyms, can be processed similarly, resulting in a muldingual dictionary. This has been checked using several Eurotra transfer dictionarien.

'This program was first implemented in Prolog for the Ndict system ([3/oksma et al, 1990]) and modified for a $E_{11-}$ rotra research group on "Reversible T'ransfer". 


\section{4s: \{homenaj honores tributo \}. \\ nl: \{exbetoon 0.1 exbewijs 0.1$\}$.}

'The current implenentation is not yet fully satiofying. Hecaus: there in no reading distinction on the Spaniah side in the Van Dale N-S (only the Duteh words in the example are marked with a reading number, e.g. 0. 1), some clusters will get mixed $1 \mathrm{p}^{10}$. L.g. Spanish fresco as adjective mean fresh and as noun fresco, though the program will currently not make this distinction

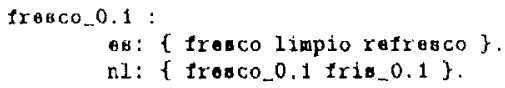

The program could of course be modified to use the granmatical information about the target word in the dictionary as reading distinguisher; the noun fresco would then never be confused with the adjective. This is undesirable in principle, however, as we do not want syntactic criteria to guide reading distinction. For instance, many adjectives in Ilonance languages have homophonous norninal counterparts, with identical nor phology and semantics. We don't want to be forced a priori to diatinguish separate readings for these two cases. Furthernore, well-known examples of category shift in translation (e.g. adverbs translating to verbs etc.) show it is impossible to attach a unique syntactic category to an equivalence class.

These presentations of synonym clusters can be very helpful to interactively improve transfer dictionaries: errork of this type can easily be detected by native epeakers of the languages (who need not know the othar language) and corrected by creating appropriate reading distinction in Spanish.

We checked the quality of the synony m clusters gener. ated from trom both Van Dale and a lívoorna SpanishDutch dictionary. The Furotra dictionary, where both source and target language items are referred to at the reading level, was converted to over 2187 clusters, 315 of which contrined more than one Sparish reading. Native speakers agreed with more than $95 \%$ of these synonym sets generated via the bilingual closure step. 'The interpretation of bilingual trauslation as synonymy is therefore correct in the vast majority of cases.

However, exceptions exist, such as the translation of the Spanish reloj, which, even though a true (and infrequent) Dutch synonym exists (viz. uurwerd (ci. English (imepiece)), more commonly translates to one of its hyponyms horloge (Eigg watch) or klok (Eng clock).

An interesting elaboration of our approach would be to extend the knowledge base by ordering the synonym clusters themselves via hyponomy ${ }^{11}$ (cf. [Cruse, 1986],

\footnotetext{
${ }^{10}$ The problem of comecting word forms to their readings las beell called the mapping problem. Of. [Byrd et al, 1987] for discussion of a method to map word foms to readings by comparing a.o. nemantic features like human of the source reading and potential target readings.

"Ihis idea is aimilar to Wordnet, a collection of nynonym sets linked via a variety of lexical relations ([Beckwirth et al., 1989]). Our approach extends this idea by adding a multilingual dimension. Worduet'n symonym suts are also related by relationy with lens dest translational consequences.
}

[Lyonk, 1977]). Client applications could then extruct translational data bared not only on synonymy but also on hyp(er)onymy. However, this is a dillicult area, where no obvious solutions exiat. It in not clesar at all which translation solution antomatic transhators should select in cases like this anyway.

A fuer this correction process the symonym clusters cun be converted to TlS format and atored in the datubase. The awsociated monolingual dictionates are then modified automatically by adding cross-reference information (via the feature semantics) from the lexical entries to the synonym chasters they are associated with.

\subsection{Creating a knowledge buse}

Symonym clusters really becone descriptions of desig nations once semantic information is added to the syuonym chusters, which is then, in a truly interlingual way, shared between synonyms. Much gemantic information from the ('retix l)utch dictionary can be moved to the synonym clusters, us well as Van Dale definitions of concepts in natural language. The latter are useful for seminutomatic interactive applications ${ }^{12}$.

The current approach can be said to implement the approach of possible bilingual lexical translation. This approach should be developed in a number of ways. Apart from the problem of translation to non-synonyms we inentioned, it is desirable to include information in the dictionary to guide the choice among possible transla. tions, in cases where there are several synonyms in the target language. Stylistic, collocational and frequency information can be of use for this purpose. This information is partly avalable from existing sources (such as ChIEX and Van Dale), und large text corpora are also obviously relevant sources of this information.

\section{A model for conversion}

Conversion or exchange of lexical data presupposes a detailed comparison of the various dictionaries, which in turn requires a careful description of the various dictionaries. Given the purpose of comparimon, the descriptions should be cast in a uniform, preferably high-level data description language. Several such languages exist, such as the Rintity-Relationship model, a tool in database design. We will use the THS formalism introduced in section 3 for this purpose.

A first step in this conparison is to convert various dictionaries to the uniform TIS format. In most NI.P fornalisms lexical entries are records or feature atructures, so this syntactic transformation is generally unproblen?atic. In passing, implicit semantic structure in the various dictionaries (e.g. Feature cooccurrence restrictions) can be rendered explicit by construcling a type hicrarchy for these systems.

On the basis of these descriptions, constraints on the? relation between lexical entries in the difterent dictionaries can be defined. These consiraints can be called

Also sece [Calzolari, 1990] for a proponal similar to ours to integrate the dictionary and the thesaurus.

"For example, loosetta incorperater an interactive reading selection facility. 
semantic, as they relate the content of the various dictionaries, and neutral as they merely pinpoint correspondences between dictionaries; they define the way dictionaries (which may be unrelated in other respects) are similar.

Constraints can be viewed as implicational and biconditional constraints (as in [van der Eijk, 1992b]), and it is possible to implement them as a complex TFS type. This type serves both as documentation of the dictionary and as conversion specification.

A conversion specification is a TFS type convERT having features for each of the dictionaries (e.g. lexic, eurotra and rosetta), and establishes the basic conversion relation between entries in the LEXIC dictionary (as derived from the sources and augmented by lexicographers) and entries in the EURotra and RosetTa dictionaries. This conversion type is structured hierarchically as well: the high-level type CorVERT has many subtypes specifying how specific subtypes (and hence subsets of the respective lexicons) of the various dictionaries are related. Disjuncts in the constraints of these types enumerate corresponding patterns described as feature structures.

An advantage is that these conversion constraints can be defined at the appropriate level of abstraction. It is in principle possible to establish relations holding for allentries as well as for an individual entry. As the conversion types are also ordered in an inheritance hierarchy, subtypes will inherit the constraints of their supertype(s).

Note the inherent declarative character of the conversion constraints: there is no notion of 'input' and 'output'. One advantage of this is that a single formalism can be used for importation, generation as well as integration of lexicons. A second advantage is that the conversion constraints can also be used to test whether two existing dictionaries are related as postulated in the conversion constraints.

Full derivability of a particular dictionary can be viewed as a special case of the general (in principle relational) scheme, where the substructure of a feature like rosetta is fully (and functionally) derivable from the substructure of another (lexic). Informally, all primitive distinctions in the target dictionary can be computed given the information in the source dictionary, i.e. the constraints define a homomorphism from the serving dictionary to the client application.

It is an empirical issue whether this derivability relation can actually be defined between two dictionaries. For newly to be created "generic" lexicons, this derivability is a design requirement. For the client dictionaries we have had to look at, creation of a generic source appeared to be a complex, but feasible, task.

Operationally, conversion proceeds as query-evaluation. Given an appropriate definition of the CONVERT type, the solutions to the following query will find all lexical entries whose canonical form is fiets in the IJEXIC database and return all corresponding further instantiations of the ROSETTA type.

These instantiations correspond to the RosetTA descriptions for this lexical entry.

$$
\left[\begin{array}{l}
\text { CONVERT } \\
\text { lexic }:\left[\begin{array}{l}
\text { ENTRY } \\
\text { canonical_form }: \text { fiets }
\end{array}\right] \\
\text { rosetta : ROSETTA }
\end{array}\right]
$$

\section{Illustration}

We will illustrate conversion using the example in [van der Eijk, 1992a] relating two familiar linguistic theories, GPSG and a unification variant of Categorial Grammar, rather than the LEXIC fragment and ROSETTA, which we actually implemented.

The categorial lexical entries have a feature subcat whose value is either a CATEGORY or a FUNCTION. The type FU:CTION has appropriate features argument, (with two features direction and category), and resull, where the result can be either a function again or a CATEGORY. Individual lexical entries are simply instances of this highly general recursive scheme. E.g. the subcat feature of a transitive verb (i.e. (MP $\backslash S$ ) / MP) has type FUNCTION, with an IP argument to the right and, recursively, a FUNCTION from a subject HP to an 5 as result.

In GPSG individual lexical entries also have a feature subcat, but its value, an integer, is used to select the corresponding context-free grammar rule for this complementation pattern.

One of the disjuncts of the constraints for the CONVERT type will then be the following. Unifying specific categorial entries into the $\mathrm{cg}$ substructure will cause the corresponding $g p s g$ feature to become instantiated.

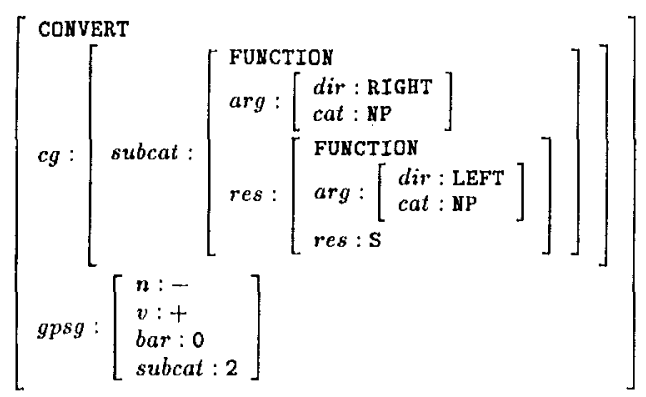

Due to the declarative character of TFS constraint evaluation, the above constraint will yield the same result whether the $c g$, the $g p s g$ or both features are instantiated.

Evidently, the example is very simplistic. The prototype conversion module we developed in our project to translate LEXIC fealure structures to RoseTta feature structures contained over 500 disjuncts $^{13}$, and this module only covered conversion of a subset of the verbs. This number is caused by the fact that conversion rules

\footnotetext{
${ }^{13}$ This number results from expansion to disjunctive normal form. The actual notation for conversion rules allows for embedded disjunctions and is, hence, much more concise.
} 
tend to become very idiosyncratic once the underlying theories of two dictionaries diverge.

\section{Conclusion}

We discussed how a multilingual lexical database can be constructed using a number of existing lexical resources and lexicography. The TFS formalism is very appropriate for the design and implementation of NLP lexicons. We showed that its hierarchical structure can be used profitably in a data entry tool which allows the lexicographer to manipulate feature structures graphically. Lexical acquisition from existing lexical resources can be combined seamlessly with lexicographic work.

The lexicon architecture we designed is an important improvement over earlier approaches: various abstraction levels and the mappings between them are defined more precisely, and the modularity is increased significantly by the separation of the knowledge base from language-specific dictionaries.

With respect to the issue of reusability, we outlined a framework for the specification of comparative description of linguistic encoding schemes. This specification can be used operationally as translation rules to convert lexical data.

\section{References}

[Beckwirth et al., 1989] Richard Beckwirth, Christiane Fellbaum, Derek Gross, and George Miller. Wordnet: A lexical database organized on psycholinguistic principles. Paper presented at the First Lexical Acquisition Workshop, IJCA189, 1989.

[Bloksma et al., 1990] Laura Bloksma, Anne van Bo]huis, I'im van der Fijk, Pius ten Hacken, Joy Herklots, Dirk Heylen, Fans Pijnenburg, Frank Sesink, AnneMarie Teeuw, Louis des Tombe, and Ton van der Wouden. Ndict: Final report. Technical report, Eurotra-NL, University of Utrecht, 1990.

[Boguraev and Briscoe, 1989] Bran Boguraev and Ted Briscoe, editors. Computational Lexicography for Natural Language Processing, London and New York, 1989. Longman.

[Byrd et al., 1987] Roy Hyrd, Nicoletta Calzolari, Martin Chodorow, Judith Klavans, Mary Neff, and Ommeya Rizk. Tools and methods for computational lexicology. Computational Linguistics, 13(3-4), 1987.

[Calzolari, 1990] Nicoletta Calzolari. The dictionary and the thesaurus can be combined. In Relational Models of the Lexicon. Martha Evens, 1990.

[Carpenter, 1990] Hob Carpenter. The logic of typed feature structures. Draft, 1990.

[Cruse, 1986] D.A. Cruse. Lexical Semantics. Cambridge University Press, 1986.

[Emele and Zajac, 1990] Martin Éruele and Rémi Zajac. 'Typed unification grammars. In Proceedings of the 19th International Conference on Computational Linguistics (COLING), 1990.
[Fokker, 1992] Jeroen Fokker. Lemming user manual. Technical Report INF/DOC-92-04, Department of Computer Science, State University of Utrecht, 1992.

[Franz, 1990] Alex Franz. A parser for HPSG. Technical report, Labotatory for Computational Linguistics, Carnegie Mellon University, 1990. No. CMU-I,CL-903 .

[Longman, 1987] Longman. Longman Dictionary of Contemporary English. Longuan House, Burnt Mill, Harlow, Essex, England, 1987. Second Edition.

[Lyons, 1977] John Lyons. Semantics. Cambridge University Press, 1977.

[McNaught, 1988] John McNaught. Computational ]exicography and computational linguistics. Lexicographica, (4), 1988.

[Picht and Draskau, 1985] Heribert Picht and Jennifer Draskau. Terminology: An Introduction. University of Surrey, 1985.

[ten Hacken, 1990] Pius ten Hacken. Reading dictinction in MT. In Proceedings of the 19th International Conference on Computational Linguistics (COLING), 1990.

[van der Eijk et al, 1991] Pim van der Eijk, Laura Bloksmi, Anne vası Bolhuis, Joy Herklots, Elly van Munster, Jeroen Fokker, Mark van der Kraan, and Angelique Geilen. Final report of the Lexic Project Phase 1. Technical report, Foundation for Language Technology, 1991.

[van der Eijk, 1992a] Pin van der Eijk. Multilingua] lexicon architecture. Working Papers in Natural Language Processing, Katholieke Universiteit Leuven, Stichting Taalechnologie Utrecht, 1992. forthcoming.

[van der Eijk, 1992b] Pim van der Eijk. Neutral dictionaries. In Cheng-Ming Guo, editor, Machine Tractable Dictionaries: Design and Construction, chapter 6 . Ablex, 1992. forthcoming.

[van Sterkenburg et al., 1982] Piet

van Sterkenburg, Willy Martin, and Bemard Al. A new Van Dale project: Bilingual dictionaries on one and the same monolingual basis. In J. Goetschalckx and L. Rolling, editors, Lexicography in the electronic age, pages 221-237. North-Holland, Amsterdam, 1982.

[Zajac, 1990] Rémi Zajac. A relational approach to translation. In Proc. 3rd Int. Conf. on Theoretical and Methodological Issues in Machine Translation of Natural Language, 1990.

[Zernik and Jacobs, 1990] Uri Zernik and Paul Jacobs. 'Tagging for learning: Collecting thematic relations from corpus. In Procedings of the 19th International Conference on Computational Linguistics (COLING), Helsinki, 1990.

[Zgusta, 1971] Ladislav Zgusta. Manual of Lexicography. Mouton, 1971. 